\title{
Satisfação de usuários com o acolhimento e classificação de risco em unidades públicas de saúde
}

Lana Aires Marques ${ }^{1}$, Flaviane Cristina Rocha César ${ }^{2}$, Livia Cristina de Resende Izidoro ${ }^{3}$, Karynne Borges Cabral ${ }^{4}$, Leidiene Ferreira Santos ${ }^{5}$, Virginia Visconde Brasil ${ }^{6}$, Lizete Malagoni de Almeida Cavalcante Oliveira ${ }^{7}$

\section{RESUMO}

O objetivo foi avaliar a satisfação dos usuários de unidades municipais de saúde que realizam Acolhimento com Avaliação e Classificação de Risco (AACR). Estudo descritivo transversal, quantitativo, realizado em quatro serviços com AACR realizado por enfermeiro, em capital do Centro-Oeste do Brasil. Dados colhidos em 2017 com 101 usuários, usando formulário com 44 perguntas objetivas sobre cinco dimensões: equipe de saúde, tempo, estrutura física, conforto e atendimento em geral. Predominaram mulheres (54,5\%), idade de 18 a 39 anos (65,3\%) e 10 a 12 anos de escolaridade (58,4\%). Mais de 2/3 estavam satisfeitos com as dimensões avaliadas, mas houve usuários indiferentes e insatisfeitos em todas. Apesar da predominância de usuários satisfeitos, é necessário repensar a organização e funcionamento dos serviços, trabalhando tanto para manter ou melhorar a qualidade do que foi avaliado positivamente, quanto para corrigir o que ainda não está bom para usuários insatisfeitos, visando a excelência do atendimento.

Descritores: Comportamento do Consumidor; Serviços Médicos de Emergência; Acolhimento; Enfermagem em Emergência.

\footnotetext{
${ }^{1}$ Acadêmica de Enfermagem da Faculdade de Enfermagem da Universidade Federal de Goiás. Bolsista do Programa de Bolsas de Iniciação Científica (PIBIC) do CNPq-UFG. Goiânia, GO, Brasil. E-mail: lanaairesmarques@hotmail.com.

${ }^{2}$ Enfermeira, Discente do Programa de Pós-Graduação em Enfermagem, nível Doutorado, da Faculdade de Enfermagem da Universidade Federal de Goiás. Professora Substituta da Universidade Federal de Jataí. Jataí, GO, Brasil. E-mail: flaviane rocha01@hotmail.com.

${ }^{3}$ Enfermeira, Mestre em Enfermagem. Discente do Programa de Pós-Graduação em Enfermagem, nível Doutorado, da Faculdade de Enfermagem da Universidade Federal de Goiás. Goiânia, GO, Brasil. E-mail: liviaresende.enf@gmail.com.

${ }^{4}$ Enfermeira, Mestre em Enfermagem. Discente do Programa de Pós-Graduação em Enfermagem, nível Doutorado, da Faculdade de Enfermagem da Universidade Federal de Goiás. Goiânia, GO, Brasil. E-mail: karynneenf26@ hotmail.com.

${ }^{5}$ Enfermeira, Doutora em Ciências da Saúde. Professora do Departamento de Enfermagem da Universidade Federal do Tocantins. Palmas, TO, Brasil. E-mail: leidienesantos@uft.edu.br.

${ }^{6}$ Enfermeira, Doutora em Enfermagem. Professora Associada da Faculdade de Enfermagem da Universidade Federal de Goiás. Goiânia, GO, Brasil. E-mail: visconde@ufg.br.

${ }^{7}$ Enfermeira, Doutora em Ciências da Saúde. Professora Titular da Faculdade de Enfermagem da Universidade Federal de Goiás. Goiânia, GO, Brasil. E-mail: lizete@ufg.br.
}

\section{Como citar esse artigo:}

Marques LA, César FCR, Izidoro LCR, Cabral KB, Santos LF, Brasil VV, et al. Satisfação de usuários com o acolhimento e classificação de risco em unidades públicas de saúde. Rev. Eletr. Enf. [Internet]. 2018 [acesso em:

];20:v20a24. Disponível em: https://doi.org/10.5216/ree.v20.50113. 


\section{INTRODUÇÃO}

O sistema de saúde brasileiro está estruturado em níveis de baixa (atenção primária), média (atenção secundária) e alta densidades tecnológicas (atenção terciária), visando garantir a integralidade do cuidado. A porta de entrada do usuário no sistema é a atenção primária, estruturada como primeiro nível de atenção para garantir atendimento das necessidades de saúde de toda a população( ${ }^{(1)}$. Entretanto, devido à falta de agilidade e baixa resolutividade dos serviços de atenção básica, a população com menor renda demonstra baixa adesão aos serviços oferecidos nesse nível de atenção, preferindo usar atendimento para suas necessidades de saúde nos Serviços de Urgência e Emergência (SUE), como alternativa para conseguir atendimento em menor prazo ${ }^{(2-3)}$.

A consequência é a superlotação dessas unidades, que passam a ser usadas como porta de entrada para o Serviço Único de Saúde (SUS). Além disso, a alta demanda impõe aos SUE o desafio de oferecer assistência de qualidade, garantindo atendimento por ordem de prioridade e não mais por ordem de chegada e sem critério clínico $^{(4)}$.

O serviço de Acolhimento com Avaliação e Classificação de Risco (AACR), proposto pela Política Nacional de Humanização (PNH), surgiu como estratégia para aprimorar o atendimento e o acesso aos usuários dos SUE. O acolhimento tem objetivo de incluir e estabelecer relação com os que buscam atendimento nos serviços de baixa, média e alta densidades tecnológicas. A avaliação com classificação de risco (ACR) objetiva conhecer as prioridades e avaliar o risco de cada usuário, seguindo protocolo previamente estabelecido e deve ser realizada por enfermeiro(5).

Juntos, acolhimento e ACR constituem ferramenta capaz de melhorar o atendimento e reduzir o tempo de espera dos usuários, com a proposta de reorganizar os SUE e inovar suas práticas gerencial e assistencial para superar os desafios do atendimento aos usuários. Dentre tais desafios, destacam-se a superlotação, a desumanização da assistência e a nova ordem dos atendimentos de acordo com o risco apresentado pelo

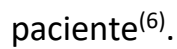

Apesar dos avanços e conquistas, a implantação do AACR nos SUE ainda está em construção, necessitando avaliações contínuas para identificar e corrigir falhas para oferecer melhorias na qualidade dos serviços disponibilizados. Ainda considerando que as mudanças políticas e organizacionais, geralmente, são propostas para melhorar os serviços ofertados aos usuários, é fundamental conhecer a opinião destes sobre a qualidade do atendimento recebido. $O$ respeito às expectativas e necessidades dos usuários e sua satisfação com o atendimento recebido nos serviços de saúde direciona o planejamento em saúde e é indispensável para repensar as práticas assistenciais e gerenciais dos serviços ${ }^{(7-8)}$. Além disso, estudos de avaliação da satisfação constituem ferramenta para a participação da comunidade que usa o serviço(9).

A avaliação rotineira dos serviços de saúde contribui para o fortalecimento do SUS e assunção do usuário como objeto central do trabalho dos profissionais ${ }^{(10)}$. A avaliação de políticas, programas e serviços de saúde possibilita a reorientação dos planejamentos, das ações e das estratégias usadas para alcançar o êxito do atendimento oferecido. Além dos aspectos quantitativos, a avaliação da qualidade de um serviço deve considerar, também, a qualidade da assistência aos usuários e a satisfação destes com o atendimento recebido ${ }^{(11)}$. Ao planejar estratégias para melhoria da qualidade do atendimento, os administradores de serviços de saúde que buscam a excelência devem considerar a percepção dos usuários sobre o atendimento recebido ${ }^{(12-15)}$. Além disso, para 
garantir a participação social no sistema de saúde pública, o usuário precisa ser visto como indivíduo capaz de avaliar e propor mudanças visando melhoria desse sistema.

Entretanto, satisfazer os usuários de um serviço requer conhecimento de suas reais necessidades, que só pode ser conseguido pela análise das informações obtidas diretamente deles. A avaliação da satisfação dos utentes é valiosa para o planejamento de ações para resolver problemas cotidianos visando melhoria da qualidade do atendimento oferecido ${ }^{(12)}$. Esse estudo objetivou avaliar a satisfação dos usuários com o serviço de AACR de unidades municipais de saúde em capital da região Centro-Oeste do Brasil.

\section{MATERIAL E MÉTODOS}

Estudo descritivo transversal, com abordagem quantitativa, realizado em quatro SUE mantidos pela Prefeitura Municipal de uma capital do Centro-Oeste do Brasil que possuem serviço de AACR realizado por enfermeiro. Estas unidades oferecem atendimento de baixa e média densidades tecnológicas a pacientes de toda a cidade, com serviços de exames diagnósticos, urgências e emergências e terapia renal substitutiva em uma das unidades.

O projeto foi aprovado pelo Comitê de Ética em Pesquisa do Hospital das Clínicas da Universidade Federal de Goiás da cidade de Goiânia-GO (pareceres 542.658 e 1.398.916), respeitando a regulamentação brasileira para pesquisas com seres humanos.

A população foi constituída por usuários dos SUE citados, com amostra por conveniência, totalizando 101 usuários. Foram incluídos indivíduos com idade igual ou superior a 18 anos, que já haviam sido atendidos no AACR naquele dia e aguardavam atendimento médico. Indivíduos manifestando algum grau de sofrimento (psicológico/emocional ou físico) ou dificuldade cognitiva registrada em prontuário foram excluídos. A participação dos usuários foi voluntária e condicionada à assinatura do Termo de Consentimento Livre e Esclarecido.

A coleta de dados foi realizada por uma pesquisadora de janeiro a junho de 2017 nas unidades onde os participantes tinham sido atendidos, usando formulário construído com base em instrumento do Ministério da Saúde para avaliação de serviços de saúde ${ }^{(16)}$, na literatura disponível e nos objetivos do estudo. A coleta ocorreu nos horários de funcionamento do AACR das unidades e após o atendimento pelo enfermeiro, com duração de 10 a 15 minutos por entrevistado.

O formulário continha 44 perguntas fechadas, cada uma com cinco opções de respostas tipo Likert (de "muito insatisfeito" a "muito satisfeito"). Além de sexo, idade e nível de escolaridade, o instrumento incluía a avaliação da satisfação em cinco dimensões: equipe de saúde, tempo, estrutura física, conforto e avaliação geral.

Os resultados da análise estatística descritiva (frequências absoluta e relativa) são apresentados em tabelas, sendo os itens que compõem cada dimensão expostos apenas descritivamente. A associação entre idade, sexo e tempo de escolaridade e a satisfação nas cinco dimensões avaliadas foi testada usando o qui-quadrado ou teste exato de Fisher, quando indicado. Foram consideradas significativas as associações com valor de $p<0,05$. 


\section{RESULTADOS}

Os resultados evidenciam uma semelhança entre o número de participantes em três dos quatro serviços investigados (entre 30,7 e 37,6\%). Dos 101 usuários incluídos, mais da metade era do sexo feminino (55; 54,5\%), tinha idade de 30 anos ou mais $(61 ; 60,4 \%)$ e tempo de escolaridade de 10 anos e mais $(77 ; 76,2 \%)$, correspondente ao ensino médio e superior (Tabela 1 ).

Tabela 1: Distribuição dos usuários de acordo com a unidade investigada, faixa etária, sexo e escolaridade. Goiânia, GO, Brasil, 2017.

\begin{tabular}{llll}
\hline Variáveis $(\mathbf{n}=\mathbf{1 0 1})$ & Categorias & $\mathbf{f}$ & \% \\
\hline \multirow{3}{*}{ Unidade investigada } & A & 31 & 30,7 \\
& B & 8 & 7,9 \\
& C & 24 & 23,8 \\
Faixa etária em anos & D & 38 & 37,6 \\
\hline \multirow{2}{*}{ Sexo } & $18-29$ & 40 & 39,6 \\
& 30 e mais & 61 & 60,4 \\
\hline \multirow{2}{*}{ Tempo de escolaridade } & feminino & 55 & 54,5 \\
& masculino & 46 & 45,5 \\
\hline
\end{tabular}

De maneira geral, mais de 2/3 dos participantes afirmaram estar "satisfeitos" e "muito satisfeitos" em todas as dimensões avaliadas e a dimensão com maior número de insatisfeitos/indiferentes $(30,7 \%)$ foi a Equipe de saúde (Tabela 2).

Tabela 2: Satisfação dos usuários com as dimensões do serviço de Acolhimento e Avaliação com Classificação de Risco em SUE municipais. Goiânia, GO, Brasil, 2017.

\begin{tabular}{llllll}
\hline & $\begin{array}{l}\text { Muito } \\
\text { insatisfeito }\end{array}$ & Insatisfeito & Indiferente & Satisfeito & Muito satisfeito \\
\cline { 2 - 6 } Dimensões & $\mathbf{f ( \% )}$ & $\mathbf{f ( \% )}$ & $\mathbf{f ( \% )}$ & $\mathbf{f ( \% )}$ & $\mathbf{f ( \% )}$ \\
\hline Equipe de saúde & $1(1,0)$ & $3(3,0)$ & $27(26,7)$ & $59(58,4)$ & $11(10,9)$ \\
Tempo & $0(0,0)$ & $5(5,0)$ & $13(12,9)$ & $67(66,3)$ & $16(15,8)$ \\
Estrutura física & $1(1,0)$ & $1(1,0)$ & $25(24,8)$ & $60(59,4)$ & $14(13,9)$ \\
Conforto & $0(0,0)$ & $2(2,0)$ & $20(19,8)$ & $74(73,3)$ & $5(5,0)$ \\
Avaliação geral & $1(1,0)$ & $4(4,0)$ & $25(24,8)$ & $59(58,4)$ & $12(11,9)$ \\
\hline
\end{tabular}

Os itens melhor avaliados no atendimento recebido da equipe de saúde estão associados à satisfação com o quantitativo de profissionais de enfermagem $(47,8 \%)$, confiança no profissional para falar sobre seu problema $(46,6 \%)$ e cuidado e detalhamento do profissional de enfermagem nos exames realizados (46,5\%).

Nessa mesma dimensão, há usuários insatisfeitos com os seguintes aspectos: gentileza dos funcionários na recepção $(15,8 \%)$, disponibilidade dos profissionais para dar as orientações necessárias $(17,8 \%)$, fornecimento de informações sobre o tempo de espera para atendimento $(20,8 \%)$, gentileza dos funcionários do serviço de AACR $(15,8 \%)$, disponibilidade dos profissionais de enfermagem para lhe atender (12,9\%), interesse dos profissionais em seu(s) problema(s) de saúde (12,9\%), paciência da equipe de enfermagem para escutar seus problemas de saúde $(13,9 \%)$, cuidado e detalhamento do enfermeiro ao Ihe examinar $(9,9 \%)$, confiança no profissional de 
enfermagem para contar seus problemas de saúde $(13,9 \%)$, confiança no profissional de enfermagem que realizou sua avaliação e classificação de risco (12,9\%) e com a classificação a ele atribuída pelo enfermeiro na avaliação de risco (9,9\%). O tempo de espera para ser atendido tanto pela recepcionista da unidade como pelo enfermeiro da classificação de risco gerou insatisfação em $11,9 \%$ dos entrevistados.

Na dimensão Estrutura física, mesmo a maioria $(73,3 \%)$ dos usuários tendo se mostrado satisfeitos, havia alguns insatisfeitos com diversos itens: conservação do prédio $(8,9 \%)$, adequação das instalações físicas da unidade $(9,9 \%)$, visibilidade e clareza das informações sobre os serviços disponíveis $(10,9 \%)$, sinalização e facilidade para encontrar o local onde precisava ir $(5,9 \%)$ e facilidade de deslocamento na unidade $(8,9 \%)$.

Quanto à dimensão Conforto, havia usuários insatisfeitos com: existência de banheiros (11,9\%), sua limpeza e conservação $(13,9 \%)$, disponibilidade de água e sabão $(18,8 \%)$ e toalha de papel $(24,8 \%)$, disponibilidade de água potável (14,9\%), conforto das cadeiras para espera (15,8\%), limpeza $(9,9 \%)$ e ventilação da sala de espera (14,9\%), e limpeza $(11,9 \%)$ e ventilação $(17,8 \%)$ do ambiente da avaliação e classificação de risco.

Nenhuma das unidades estudadas dispunha de maca para exame dos pacientes na sala de classificação de risco e eles são examinados sentados em uma cadeira. Mesmo assim, havia usuários satisfeitos com a existência de maca(s) com colchonete para exame dos pacientes $(29,7 \%)$, lençol descartável para o colchonete $(26,7 \%)$ e limpeza da maca e colchonete (24,8\%).

$\mathrm{Na}$ avaliação geral do serviço e do atendimento recebido, os usuários mostraram-se insatisfeitos com os serviços oferecidos na unidade (13,9\%), organização e funcionamento da unidade $(8,9 \%)$, atendimento de forma geral (10,9\%), na recepção (11,9\%) e na classificação de risco (10,9\%). Merece destaque o número de usuários $(16,8 \%)$ "muito insatisfeitos" e "insatisfeitos" com o sistema de classificação de risco para o atendimento nos SUE. Também houve participantes insatisfeitos na avaliação geral do atendimento recebido no AACR $(10,9 \%)$ e com o serviço em geral (14,9\%), desde a recepção até o atendimento pelo enfermeiro.

Entre as variáveis sociodemográficas investigadas, apenas a idade foi estatisticamente associada às dimensões Estrutura Física $(p=0,031)$ e Conforto $(p=0,020)$.

\section{DISCUSSÃO}

O ACCR é uma estratégia de atendimento proposta para amparar gestores, trabalhadores e usuários dos serviços de saúde, favorecendo a equidade, permitindo agilidade no atendimento às urgências e emergências, evitando superlotação de serviços e garantindo prioridade de atendimento para os casos mais graves ou com maior risco. Isso o torna crucial para o planejamento e execução de ações que influenciam no atendimento resolutivo e de boa qualidade. Entretanto, a realidade nos SUE demonstra que ainda há despreparo da equipe multiprofissional em algumas situações, dificuldades no fluxo de atendimento, inadequação da estrutura física e escassez de recursos materiais que podem influenciar na satisfação do usuário com o atendimento.

Nesse cenário, a avaliação da satisfação dos usuários fornece importantes parâmetros para a (re)organização desses serviços. O presente estudo avaliou dimensões em que administradores e enfermeiros possuem gerência: equipe de saúde, tempo, estrutura física, conforto e funcionamento do serviço. Por dificuldade de acesso aos pacientes após o atendimento médico, essa dimensão não foi avaliada. 
A faixa etária e gênero predominantes coincidem com essa reconhecida maioria na busca por atendimentos de saúde. Entretanto, o tempo de escolaridade, que no presente estudo foi majoritariamente de 10 anos e mais, é maior que o encontrado em outros estudos ${ }^{(9-10)}$.

Apesar do número notadamente maior de usuários satisfeitos com a todas as dimensões avaliadas, há muitos indiferentes e insatisfeitos em todas elas. Esses resultados adquirem significado tanto para reforço positivo do que está sendo efetivo, como para a necessidade de maior atenção àqueles aspectos que ainda podem melhorar e se aproximar do ideal de satisfação para todos os usuários.

Mesmo considerando o alto percentual de participantes satisfeitos em todas as dimensões avaliadas e que a satisfação é um processo dinâmico que pode sofrer influência da idade, expectativas, antecedentes, valores, condição física, escolaridade e outros ${ }^{(17)}$, ainda há muito a ser feito em busca da satisfação de todos os usuários. Além disso, altos índices de satisfação podem estar relacionados com menores expectativas dos utentes ${ }^{(9)}$, muito comuns em grande parte dos usuários do SUS, onde ainda se observa certo desconhecimento acerca de seus direitos à saúde(18). Vale lembrar que expectativas baixas são mais facilmente atingidas, não estando necessariamente atreladas à qualidade do serviço prestado; portanto, nessa situação, é pouco significativo o alto nível de satisfação observado ${ }^{(9)}$.

Alto nível de satisfação associado com baixa escolaridade já foi observado em outras investigações ${ }^{(19-20)}$, sugerindo que a formação escolar contribui para que os usuários desenvolvam sua capacidade de crítica em relação aos serviços recebidos e, consequentemente, sejam mais exigentes quanto à sua qualidade. Quanto menor a escolaridade, maior a condescendência dos usuários com a qualidade dos serviços de saúde e maior a sua satisfação com eles ${ }^{(9)}$. O mesmo pode ser afirmado quanto à idade, que costuma apresentar uma relação inversamente proporcional à satisfação com os serviços, isto é, quanto mais jovem o usuário, menor sua complacência e, consequentemente, maior a insatisfação(10).

Quanto à equipe de saúde, quase metade dos usuários mostraram satisfação com o quantitativo de profissionais de enfermagem, com a confiança para falar com eles sobre seu problema de saúde e com o cuidado do enfermeiro no seu exame. Pode parecer um bom percentual, mas é preocupante quando se considera os dois últimos itens. O esperado é que todos confiem na atuação dos profissionais. Portanto, a equipe de enfermagem deve atentar para sua relação com os clientes e para a forma como está desempenhando sua função, buscando os pontos frágeis e procurando novas estratégias para melhorar a confiança em seu desempenho profissional.

Embora o trabalho dos profissionais geralmente seja o item melhor avaliado pelos usuários em muitos trabalhos $^{(9-10,21)}$, ainda exige investimento na qualificação desses profissionais para melhorar seu desempenho, já que a avaliação da qualidade de um serviço sofre influência direta da maneira como os cuidados técnicos são realizados e recebidos ${ }^{(21)}$. A capacidade dos profissionais para acolher os usuários, dar orientações necessárias, esclarecer dúvidas e despertar sua confiança são determinantes da qualidade do serviço e merecem ênfase.

Nesse sentido, os resultados desse estudo indicam necessidade de treinamento e atualização dos profissionais que atuam nesses serviços para que reflitam sobre a importância da gentileza com os usuários, disponibilidade para oferecer as informações necessárias, interesse em seus problemas de saúde, paciência para ouvi-los, cuidado ao examiná-los e competência para realizar seu trabalho, itens onde houve mais usuários insatisfeitos e que estão associados à satisfação da maioria dos usuários ${ }^{(10)}$. 
Em situações de emergência, as ações do enfermeiro devem ser pautadas na eficiência e resolutividade, sem desvalorizar a subjetividade do ser humano. É necessário refletir sobre o amplo significado do acolhimento e a atuação do profissional nos espaços de prestação do cuidado para que o usuário se sinta realmente acolhido. Esse processo conduz ao verdadeiro encontro entre profissional de saúde e usuário, promovendo sua aproximação principalmente pela capacidade de escuta para compreender as necessidades de saúde do indivíduo(6).

A qualidade da relação profissionais de saúde - usuários revela-se pela humanização das ações. Em urgência e emergência, enfermeiros devem pautar suas condutas e ações pela humanização da assistência, tornando-se mais solidários e humanos, enxergando o sofrimento alheio e dando real importância ao acolhimento que antecede a classificação de risco ${ }^{(19-22)}$. Este processo melhora a qualidade da assistência e contribui para a satisfação dos usuários.

O tempo de espera para atendimento é um determinante importante da satisfação dos usuários e, assim, a insatisfação com o tempo de espera para ser atendido tanto na recepção quanto na classificação de risco é comum $^{(10,23)}$. A alta demanda nos SUE visando rápida resolutividade para problemas de saúde que poderiam ser resolvidos em outros pontos da rede de atenção à saúde resulta em superlotação do serviço, comprometendo a qualidade do atendimento e a imediata resolução do problema ${ }^{(16)}$. Some-se a isso a histórica escassez de recursos materiais e de profissionais na maioria dos serviços públicos de saúde e está desenhado o cenário ideal para determinar insatisfação dos usuários com o tempo gasto no atendimento.

$\mathrm{Na}$ Estrutura física dos SUE, a insatisfação de usuários está relacionada tanto com a conservação do prédio e adequação das instalações físicas, como com outros aspectos que interferem em sua movimentação dentro do serviço, como visibilidade e clareza das informações sobre os serviços que a unidade oferece, sinalização sobre a localização desses serviços e facilidade para deslocamento dentro da unidade. Embora alguns itens que produzem insatisfação dos usuários sejam de mais difícil resolução, por demandarem verbas para reformas e ou adaptação das instalações, há outros em que é possível intervir mais facilmente, como é o caso da clareza das informações e sinalização dentro da unidade.

O Ministério da Saúde reconhece que há muitas unidades de saúde cuja estrutura física é inadequada e frequentemente improvisada, prejudicando a qualidade dos serviços ${ }^{(6)}$. Consequentemente, a satisfação dos usuários com as condições oferecidas nos serviços também deixa a desejar ${ }^{(10)}$. A implantação do AACR como forma de melhorar o atendimento e garantir mais funcionalidade e segurança aos usuários requer modificações na estrutura física e adequação do ambiente, com número suficiente de salas e adequada sinalização. Recomendase, ainda, a existência de salas na parte externa, para consulta de enfermagem, aferição de sinais vitais e sala de espera para usuários cuja classificação tenha sido verde ou azul(24).

No presente estudo, a inadequação da Estrutura física gerou insatisfação de muitos participantes em itens estruturais, como os banheiros para usuários e em outros que dependem de aspectos gerenciais e ou recursos financeiros para sua disponibilização, como água, sabão e toalha de papel nos banheiros, água potável, número e conforto das cadeiras, limpeza e ventilação da recepção e da sala de classificação de risco. A quantidade e conforto das cadeiras, bem como a limpeza e temperatura da unidade também geraram insatisfação dos usuários em estudo realizado em Pernambuco ${ }^{(10)}$. Possuir idade igual ou superior a 30 anos foi significativamente associado à 
satisfação com a Estrutura física e com o Conforto, sugerindo que indivíduos mais jovens são mais exigentes com relação a estes itens ${ }^{(10,25)}$.

Quanto à avaliação do serviço em geral, os usuários estavam insatisfeitos tanto com os serviços oferecidos quanto com a organização e funcionamento da unidade, mas o que merece destaque nesse sentido é sua insatisfação com o atendimento recebido de forma geral, na recepção e na classificação de risco. Tais itens independem de investimentos financeiros, mas sim e exclusivamente de mudança na atitude dos profissionais de saúde que realizam o serviço. As alterações necessárias devem começar pela reorganização do trabalho com adequação dos fluxos internos ${ }^{(9)}$, mas precisam incluir o incentivo à reflexão dos profissionais sobre sua práxis e sobre a importância do usuário e de sua satisfação para a qualidade do serviço, de maneira a garantir seu acolhimento e atendimento a contento na unidade.

A limitação deste estudo é relacionada a ausência de participação de usuários classificados com as cores amarela e vermelha, pelo grau de sofrimento demonstrado e pela dificuldade em localizá-los após o atendimento médico. Isso pode ter produzido um viés nos resultados, especialmente, naqueles relacionados à satisfação com o sistema de classificação de risco para o atendimento das urgências e emergências. Apesar das limitações, os resultados fornecem importantes informações sobre a opinião dos usuários atendidos nos SUE municipais e devem ser considerados na sua avaliação, pois podem contribuir para melhorar a qualidade dos atendimentos dispensados à comunidade.

\section{CONCLUSÃO}

Os resultados mostram a maioria dos usuários satisfeita com todas as dimensões avaliadas: Equipe de saúde, Tempo, Estrutura física, Conforto e Atendimento em geral nos SUE investigados. Destacam-se, aqui, a confiança nos profissionais para falarem sobre seus problemas e em sua competência para atende-los. Vale enfatizar que mesmo dimensões com as quais os usuários estavam majoritariamente satisfeitos precisam ser consideradas, visando a manutenção e ou melhoria de sua qualidade e modificação do que falta para satisfazer usuários até então insatisfeitos.

Entretanto, há itens em cada dimensão avaliada com os quais havia participantes insatisfeitos, indicando que as unidades ainda precisam melhorar sua organização e funcionamento para se aproximarem da qualidade almejada. Muitos aspectos são facilmente modificáveis, dependentes quase exclusivamente da mudança de atitude dos profissionais e que precisam ser contemplados para que o processo de acolhimento seja implementado em sua totalidade. Na dimensão Equipe de saúde, eles incluem gentileza, disponibilidade para fornecer informações/orientações necessárias, interesse nos problemas de saúde do usuário e paciência para ouvi-los com atenção. Nas demais dimensões, a solução de muitos dos problemas que geram insatisfação dos usuários depende essencialmente da disponibilidade de recursos financeiros e gerência, tais como estrutura e adequação do espaço físico do serviço, móveis, materiais e equipamentos, limpeza e ventilação local.

A realização de avaliações regulares da satisfação dos usuários e sua utilização como norteadora do planejamento e reorganização é um caminho para que os serviços e profissionais de saúde aproximem-se do ideal de humanização da atenção à saúde. Por isso, deve ser incorporada na filosofia de trabalho dos gestores e 
administradores para promover a transformação das práticas profissionais, onde prevaleçam o respeito e o acolhimento ao usuário.

\section{REFERÊNCIAS}

1. Ministério da Saúde. Portaria no 4.279, de 30 de dezembro de 2010. Estabelece diretrizes para a organização da Rede de Atenção à Saúde no âmbito do Sistema Único de saúde (SUS). Brasília: Ministério da Saúde; 2010 [cited 2017 June 18]. Available from: http://conselho.saude.gov.br/ultimas noticias/2011/img/07 jan portaria4279 301210.pdf.

2. Azevedo ALM, Costa AM. A estreita porta de entrada do Sistema Unico de Saude (SUS): uma avaliacao do acesso na Estrategia de Saude da Familia. Interface (Botucatu) [Internet]. 2010 [cited 2017 Aug. 16]; 14(35):797-810. Available from:

http://www.scielo.br/pdf/icse/v14n35/3010.pdf.

3. Feijó VBER, Cordoni Junior L, Souza RKT, Dias AO. Análise da demanda atendida em unidade de urgência com classificação de risco. Saúde Debate [Internet]. 2015 [cited 2017 June 15]; 39(106):627-36. Available from:

http://www.scielo.br/pdf/sdeb/v39n106/0103-1104-sdeb-39-106-00627.pdf.

4. Oliveira GN, Vancini-Campanharo CR, Okuno MFP, Batista REA. Acolhimento com avaliação e classificação de risco: concordância entre os enfermeiros e o protocolo institucional. Rev Latino-Am Enfermagem [Internet]. 2013 [cited 2017 Aug. 2]; 21(2). Available from: http://www.scielo.br/pdf/rlae/v21n2/pt 0104-1169-rlae-21-02-0500.pdf.

5. Rates HF, Alves M, Cavalcante RB. Acolhimento com classificação de risco: que lugar é esse? Enferm Foco [Internet]. 2016 [cited 2017 May 22]; 7(2):52-6. Available from: http://revista.portalcofen.gov.br/index.php/enfermagem/article/download/795/320.

6. Ministério da Saúde. Secretaria de Atenção à Saúde. Política Nacional de Humanização da Atenção e Gestão do SUS. Acolhimento e classificação de risco nos serviços de urgência. Brasília: Ministério da Saúde; 2009 [cited 2017 Aug. 13]. Available from:

http://bvsms.saude.gov.br/bvs/publicacoes/acolhimento classificaao risco servico urgencia.pdf.

7. Fréz AR, Nobre MIRS. Satisfação dos usuários dos serviços ambulatoriais de fisioterapia da rede pública. Fisioter Mov [Internet]. 2011 [cited 2017 July 17]; 24(3):419-28. Available from: http://www.scielo.br/pdf/fm/v24n3/06.

8. Pinto SAG, Silva EMVB. Qualidade e satisfação com o serviço de urgência pediátrica: a perspectiva dos acompanhantes das crianças. Rev Enf Ref [Internet]. 2013 [cited 2017 Sep. 10]; serllI(9):95-103. Available from:

http://www.scielo.mec.pt/pdf/ref/vserllIn9/serllIn9a10.pdf.

9. Brandão ALRBS, Giovanella L, Campos CEA. Avaliação da atenção básica pela perspectiva dos usuários: adaptação do instrumento EUROPEP para grandes centros urbanos brasileiros. Ciência \& Saúde Coletiva [Internet]. 2013 [cited 2017 Sep. 10];18(1):103-14. Available from: http://www.scielo.br/pdf/csc/v18n1/12.pdf.

10. Santiago RF, Mendes ACG, Miranda GMD, Duarte PO, Furtado BMASM, Souza WV. Qualidade do atendimento nas Unidades de Saúde da Família no município de Recife: a percepção do usuário. Ciência \& Saúde Coletiva [Internet]. 2013 [cited 2017 Aug. 3]; 18(1):35-44. Available from: http://www.scielo.br/pdf/csc/v18n1/05.pdf.

11. Corrêa ÁCP, Ferreira F, Cruz GSP, Pedrosa ICF. Acesso a serviços de saúde: olhar de usuários de uma unidade de saúde da família. Rev Gaúcha Enferm [Internet]. 2011 [cited 2017 June 3]; 32(3):451-7. Available from:

http://www.scielo.br/pdf/rgenf/v32n3/03.pdf.

12. Al-Abri R, Al-Balushi A. Patient satisfaction survey as a tool towards quality improvement. Oman Mes J. 2014 [cited 2018 June 12]; 29(1):3-7. Available from: https://www.ncbi.nlm.nih.gov/pmc/articles/PMC3910415/pdf/OMJ-D-13-00247.pdf.

13. Ahmed F, Burt J, Roland M. Measuring patient experience: concepts and methods. Patient. 2014 [cited 2018 June 12];7:235-41. Available from: https://link.springer.com/content/pdf/10.1007\%2Fs40271-014-0060-5.pdf.

14. Price R, Elliott M, Zaslavsky A, Hays R, Lehrman W, Rybowski L, et al. Examining the role of patient experience surveys in measuring healthcare quality. Med. Care Res. Rev. 2014 [cited 2018 June 12];71:522-554. Available from:

https://www.ncbi.nlm.nih.gov/pmc/articles/PMC4349195/pdf/nihms654075.pdf.

15. Brandão GL, Campos CEA. Avaliação da atenção básica pela perspectiva dos usuários: adaptação do instrumento EUROPEP para grandes centros urbanos brasileiros. Cien Saude Colet. 2013 [cited 2018 June 12];18(1):103- 114. Available from:

http://www.scielo.br/pdf/csc/v22n6/1413-8123-csc-22-06-1829.pdf.

16. Ministério da Saúde. Secretaria Executiva. Departamento de Regulação, Avaliação e Controle de Sistemas. PNASS: Programa Nacional de Avaliação de Serviços de Saúde. Brasília: Ministério da Saúde; 2015 [cited 2017 June 29]. Available from:

http://bvsms.saude.gov.br/bvs/publicacoes/pnass programa nacional avaliacao servicos.pdf.

17. Kelarijani SEJ, Jamshidi R, Heidarian AR, Khorshidi M. Evaluation of factors influencing patient satisfaction in social security hospitals in Mazandaran province, North of Iran. Caspian J Intern Med [Internet]. 2014 [cited 2017 Aug. 2]; 5(4):232-4. Available from: https://www.ncbi.nlm.nih.gov/pmc/articles/PMC4247488/pdf/cjim-5-232.pdf.

18. Silva Junior GB, Dias ER. Avaliação da satisfação dos usuários de um serviço de saúde público-privado no nordeste do Brasil e a judicialização da saúde. R Dir sanit [Internet]. 2016 [cited 2018 June 12];17(2):13-29. Available from:

http://www.revistas.usp.br/rdisan/article/download/122303/119041. 
19. Guedes MVC, Henriques ACPT, Lima MMN. Acolhimento em um serviço de emergência: percepção dos usuários. Rev Bras Enferm [Internet]. 2013 [cited 2017 July 23]; 66(1):31-7. Available from:

http://www.scielo.br/readcube/epdf.php?doi=10.1590/S0034-71672013000100005\&pid=S0034-

71672013000100005\&pdf path=reben/v66n1/v66n1a05.pdf\&lang=pt.

20. Levandovski PF, Lima MADS, Acosta AM. Patient satisfaction with nursing care in an emergency service. Invest educ enferm [Internet]. 2015 [cited 2017 June 28]; 33(3):473-81. Available from: http://www.scielo.org.co/pdf/iee/v33n3/v33n3a11.pdf.

21. Arakawa AM, Lopes-Herrera SA, Caldana ML, Tomita NE. Percepção dos usuários do sus: expectativa e satisfação do atendimento na Estratégia de Saúde da Família. Rev CEFAC [Internet]. 2012 [cited 2017 Oct. 18]; 44(6):1108-14. Available from: http://www.scielo.br/pdf/rcefac/v14n6/71-11.pdf.

22. Freitas FFB, Matos GSS, Cavalcante AC, Pinheiro MBGN, Sousa MNA. O papel do enfermeiro no serviço de Acolhimento e Classificação de Risco no setor de urgência e emergência. Rev Interdisc em Saúde [Internet]. 2015 [cited 2017 June 28]; 2(3):31433. Available from: http://interdisciplinaremsaude.com.br/Volume_5/Trabalho_05.pdf.

23. Oliveira JLC, Gatti AP, Barreto MS, Bellucci Júnior JA, Góes HLdF, Matsuda LM. Acolhimento com classificação de risco: percepções de usuários de uma unidade de pronto atendimento. Texto contexto - enferm [Internet]. 2017 [cited 2017 Oct. 18]; 26(1):e090014. Available from: http://www.scielo.br/pdf/tce/v26n1/pt 0104-0707-tce-26-01-0960014.pdf.

24. Arain M, Nicholl J, Campbell M. Patients' experience and satisfaction with GP led walk-in centres in the UK; a cross sectional study. BMC Health Serv Res. 2013 [cited 2018 June 12]; 13:142. Available from:

https://bmchealthservres.biomedcentral.com/track/pdf/10.1186/1472-6963-13-142.

25. Protasio APL, Gomes LB, Machado LS, Valença AMG. Satisfação do usuário da Atenção Básica em Saúde por regiões do Brasil: 1o ciclo de avaliação externa do PMAQ-AB. Ciência \& Saúde Coletiva [Internet]. 2017 [cited 2017 Nov. 17]; 22(6):1829-44. Available from: https://scielosp.org/pdf/csc/2017.v22n6/1829-1844/pt. 